\title{
Interactive Urban Maps for People with Autism Spectrum Disorder
}

$\begin{array}{ll}\text { Amon Rapp } & \text { Maurizio Tirassa } \\ \text { Federica Cena } & \text { University of Torino } \\ \text { Guido Boella } & \text { Via Verdi, 10, Torino } \\ \text { Alessio Antonini } & \text { ICxT Innovation Center } \\ \text { Alessia Calafiore } & \text { Lungo Dora Siena, 100, Torino } \\ \text { Stefania Buccoliero } & \text { maurizio.tirassa@unito.it } \\ \text { University of Torino } & \\ \text { C.so Svizzera, 185 Torino } & \text { Roberto Keller } \\ \text { ICxT Innovation Center } & \text { Romina Castaldo } \\ \text { Lungo Dora Siena, 100, Torino } & \text { Stefania Brighenti } \\ \text { amon.rapp@gmail.com } & \text { ASL 2 of the City of Torino - Clinic } \\ \text { cena@di.unito.it } & \text { for adult ASD patients (Piemonte } \\ \text { guido.boella@unito.it } & \text { Region pilot center) } \\ \text { calafiore@di.unito.it } & \text { rokel2003@libero.it } \\ \text { antonini@di.unito.it } & \text { romina.castaldo@gmail.com } \\ \text { stefania.buccoliero@gmail.com } & \text { stefania.brighenti@hotmail.it }\end{array}$

Permission to make digital or hard copies of part or all of this work for personal or classroom use is granted without fee provided that copies are not made or distributed for profit or commercial advantage and that copies bear this notice and the full citation on the first page. Copyrights for third-party components of this work must be honored. For all other uses, contact the Owner/Author.

uses, contact the Owner/Author.
Copyright is held by the owner/author(s).

CHI'17 Extended Abstracts, May 06-11, 2017, Denver, CO, USA

ACM $978-1-4503-4656-6 / 17 / 05$.

http://dx.doi.org/10.1145/3027063.3053145

\begin{abstract}
This paper outlines the roadmap and the preliminary results of an interactive systems aimed at providing interactive urban maps tailored to people with Autism Spectrum Disorder (ASD). It aims at involving ASDs in the design process to produce personalized services that can support their daily movements and task management, improving their autonomy and sense of agency.
\end{abstract}

\section{Author Keywords}

Autism; cognitive urbanism; maps.

\section{ACM Classification Keywords}

H.5.m. Information interfaces and presentation (e.g., $\mathrm{HCI}$ ): Miscellaneous.

\section{Introduction}

Cognitive urbanism [10] studies how the characteristics of human cognition and the features of the urban environment interact in building a subjective spatial representation of urban areas, volumes, routes, and landmarks. Belonging to different communities, taking part to specific social practices, as well as having diverse cognitive skills, influence such representation and consequently how the city is lived. 
This paper outlines the roadmap and the preliminary results of a project aimed at helping people with Autism Spectrum Disorder (ASD) move and live within cities by means of interactive personalized maps. The project is inscribed in the cognitive urbanism theoretical background, since it assumes that people with different cognitive (dis)abilities perceive, use, and live the urban spaces differently. Autism is becoming a health crisis in United States, with 1 in 68 children affected by ASD today [4]. Studies in North America, Asia, and Europe have identified people with ASD with an average prevalence of between $1 \%$ and $2 \%$ [2], while research on the economic costs of autism was $\$ 268$ billion in 2015 in the United States only, with an expectation of costs rising to $\$ 461$ billion in 2025 [10].

While there is no cure for ASD, its symptoms improve with treatment and support. This project aims at helping individuals with ASD improve their autonomy and their independency in moving across the city in which they live, managing their daily tasks, and interacting with other people. Especially those ASD subjects who are currently nearly capable of moving alone across urban environments could benefit of this project in terms of increased autonomy. Others, more independent subjects, could also benefit from a reduction of the need of continuous assistance by caregivers and social operators, and possibly a decrease of the need of behavioral therapies.

ASD is characterized by problems or peculiarities in the domains of social interaction, communication, attention, and practical skills $[9,10]$. Impacts of such symptoms range from mild to disabling, affecting the individual's quality of life and that of her family. More specifically, ASD is marked by a tendency to withdrawa from social relationships and what is called "weak central coherence" (that is a difficulty in the perception of Gestalts as opposed to a sum of unrelated details)

[8]. These peculiarities imply the need to find reassurance in repetitious routines, making hard to manage unexpected events. Such characteristics may lead to peculiar modalities of spatial representation and the need of tailored supports for orientation and movements in the urban environment.

The project has three mains objectives: i) to understand how individuals with ASD represent and orient themselves in urban spaces and what kinds of "spatial needs" they have; ii) to design and develop interactive urban maps to practically support people with ASD in their daily movements and tasks within urban environments; iii) to iteratively evaluate the technological intervention in a real context of use where people with ASD can use the designed interactive maps in their everyday life.

\section{Background}

Today the most common solution for ASD treatment is behavioral intervention [3]. To this aim, technology can prove an optimal means since it does not have the emotional manifestations that characterize human relationships, which may be perceived as confusing and anxiety-inducing in ASDs [6]. However, technological supports for ASD therapy mostly only translate behavioral intervention tools [12]. Despite their therapeutic successes [3], such interventions aim to treat deficits in artificial contexts, removed from the real environments where the skills supported should be actually enacted. As a twofold result, such techniques can modify behaviors through trainings that are irrelevant to the overall improvement of the individual's 
quality of life, or, even in cases where addressing specific deficits is warranted, the separation from natural contexts of daily living might make the transfer of improvements gained to the real world difficult [12].

The project aims at addressing these issues by providing not a technological intervention, but a technological support embedded in daily life: by designing tools to be used in everyday situations, it attempts to go beyond the limitations of behavioral interventions. Besides this ecological perspective, the project points to a specific set of problems, i.e. orientation and movements in urban spaces, which have received almost no attention in the current literature. Technological tools for autism mainly support social and cognitive skills of people with ASD (especially children): for example, they try to modify and improve the core deficit of social cognition, helping children acquire social and academic skills [1]. When they attempt to design "enhanced spaces", they aim at exercising cognitive, emotional, motor, and social skills [5]. There is no projects, to our knowledge, specifically targeting spatial and orientation abilities in urban environments of individuals with ASD.

Therefore, the novelty of this project is threefold: i) Current technological employed in ASD mainly reproduce artificial behavioral interventions, while it aims at embedding the technological support in the everyday activities by providing a practical tool that can be used for moving in urban spaces; ii) Current ICT tools support ASD individuals (especially children) in improving social relationships or completing cognitive tasks, while we want to help them (especially adults) in orienting and navigating in the environment; iii) Current maps to increase accessibility to urban spaces are focused on physical disabilities, while we target cognitive ones.

\section{Method}

To reach the project's objectives we employ different qualitative and quantitative research techniques.

Design of the solution basically follows a user-centered design approach, where people with ASD are involved since the beginning of the design process to gain user requirements, as well as to assess the acceptability and effectiveness of the developed solution.

In particular, in the first stage of the project, we employ qualitative interviews and cognitive maps to collect the spatial and orientation needs of individuals with ASD. Interviews aim to explore their daily routines, their patterns of movements, their city landmarks, their technology use, and their explicit requests of support. We want to give an answer to questions like: What do people with autism currently do when navigating cities? How do they circumnavigate when there are unfamiliar issues or when they encounter a problem? Cognitive maps are mental representations of the space: basically participants are invited to draw a sketch of the city in which they live positioning themselves, as well as significant landmarks and routes within it. They are used to understand how ASD individuals represent urban environments.

Moreover, we invite them to use digital map services (e.g. Google Maps) to understand how they use such services and whether they encounter specific issues with them.

In the second stage of the project, instead, we will use Participatory Design (PD) techniques, a method to involve users in the design process, which is 
increasingly used in the field of technology for autism. Although the social and communication problems related to ASD may suggest that PD is counterindicated, various techniques have been developed for engaging autistic people in design [7]. We will involve people with ASD in the design of the interactive maps, confronting our design decisions with their needs and desires, and incorporating their feedback in our designs.

In the third stage, prototypes will be developed through iterative cycles of design-evaluation-redesign: assessments will be conducted within real-contexts of use to ensure acceptability, usability and effectiveness of the solution. A final 3-months field trial, where people with ASD can use the interactive maps in their daily activities and movements around the city, will provide an ecological assessment (related to real activities in real environments, and not to tasks assigned in the context of an artificial laboratory) of the designed solution. We will employ observation and inquiry techniques. Moreover, we will provide pre/during/post intervention psychological and neuropsychological tests to assess improvements in individuals' quality of life and in their cognitive skills, sense of autonomy, self-efficacy, etc.

\section{Preliminary results}

Until now we interviewed six individuals with ASD. Interviews were carried out by a HCI researcher and a clinical psychologist specializing in ASD. Each interview lasts about 45 minutes; participants are asked to recount their daily habits in terms of movements, use of transportation means, daily task management.

Participants are also requested to draw cognitive maps of the city in which they lived as well as of their neighborhood, pointing relevant landmarks and explaining the routes which they usually follow. Moreover, they are asked to use Google Maps and are observed during the interaction with it. Interviews were audio-recorded and then transcribed verbatim. Data are analyzed through a thematic analysis. The participants up to now were screened and recruited by ASL 2 of City of Torino, Italy - Clinic for adult ASD patients (Piemonte Region pilot center). They were all mid-functioning autistic individuals (i.e. they presented also a moderate impaired cognitive functioning and were slightly below normal mental functioning). The next step will be to involve high-functioning autistic individuals and Asperger individuals to explore whether they present different spatial representation modalities and have different needs. Final aim of the project is to design a solution that can satisfy the needs of both mid and high-functioning/Asperger individuals.

Preliminary results reveal that mid-functioning autistic individuals have extremely fixed routines in terms of daily movements. Breakdown from such routines may yield anxiety and the incapability of managing the unexpected event. Most of them are autonomous only in moving between home and close and well-known places (e.g. specific shops or the gyms). For all the other movements they go with their caregivers or tutors.

Moreover, they have a reduced capacity of extracting a Gestalt from a collection of details: they fix on specific particulars, with the tendency of losing the vision of the whole. They also present a simplified representation of the city. While they are able to recognize and use common map services like Google Maps, they express a 
need of more schematic depictions of the urban environment.

Finally, they express a need to receive support not only about managing unexpected events (such as the deviation of a bus line) but also about how to socially behave in specific situations, since they lack the "social competence" necessary to carry out most everyday interactions. Other needs are related to the opportunity of visiting new places that nevertheless present "secure" characteristics, such as being scarcely crowded, or embedded within natural contexts (e.g. parks). Features of such places mostly depend on individual preferences, since each appears to have idiosyncratic "passions" for specific kinds of place.

Based on these preliminary findings we started envisioning some features that might be designed to satisfy the needs of ASD people. The first functionality is the agenda-map. It could support daily movements (home-school, home-relatives, etc.) by allowing the planning of routes and providing tailored help to cope with routine breakdowns (e.g. if the bus line they commonly take is canceled for the day the map may provide suggestions about how to solve the problem, or a communication channel may be open with a caregiver who can guide her to the target place). Maps could be personalized on the basis of the users' habits and cognitive abilities.

The second feature is the crowdsensed map. It could be populated with comments, reviews, trails by people with ASD, caregivers, and anyone interested in contributing to the service. It might suggest places, routes, and activities (e.g. not crowded, quiet places) which could make more comfortable the users' daily life both in their home city and in tourist locations.

Moreover, they could be populated with "scripts" related to how "correctly" socially interact within certain contexts: such scripts could be provided by therapists and caregivers, contributing to create a sort of contextaware recommender system that gives geo-localized advises about how to conduct a social interaction in a specific "social" place (e.g. all the steps that must be followed when entering in a post office)

While the "agenda-map" aims to help ASD subjects during their daily living, the crowdsensed map also attempts to empower their sense of agency, enabling them to contribute to the wellness of the city in which they live (e.g. making it more accessible), as well as to be recognized for the value that they can bring to their community. This could make their voice heard, giving them the possibility of emphasizing their views of the issues and opportunities offered by the city, as well as recommending and advertising places tailored to them.

Such preliminary general features could be changed in relation both to the specific needs discovered during the further interviews with high functioning ASD and Asperger individuals and to the feedback gathered during the participatory design sessions performed in the design stage.

The implementation of this solution will be based on FirstLife (http://www.firstlife.org), a social network based on interactive maps. FirstLife is a flexible platform that can be adapted to different aims. Its architecture is composed of an interactive geographical map-based interface as a frontend and a backend to manage and search geographical data. It allows to insert and manage different kinds of Points of Interest 
(POIs), and supports crowdsourced data collection. The platform also offers social networking functionalities like messaging and group management.

\section{Conclusion}

This project represents a first step towards making our cities accessible to individuals with cognitive disabilities. It aims at being expandable to other such conditions: persons with Alzheimer's disease, after effects of head injury, and spatial agnosia, for example, could greatly benefit from interactive maps tailored to their specific orientation needs and spatial representation modalities.

\section{Acknowledgments}

This project is funded by the ICxT Innovation Center.

\section{References}

1. Sofiane Boucenna, Antonio Narzisi, Elodie Tilmont, Filippo Muratori, Giovanni Pioggia, David Cohen, Mohamed Chetouani. 2014. Interactive Technologies for Autistic Children: A Review. Cognitive Computation, 6, 722-740.

2. Christensen D. L,. Baio J., Van Naarden Braun K, et al. Prevalence and characteristics of autism spectrum disorder among children aged 8 yearsAutism and Developmental Disabilities Monitoring Network, 11 sites, United States, 2012. MMWR Surveill Summ 2016;65(No. SS-3): 1 -23.

3. Geraldine Dawson, and Karen Burner. 2011. Behavioral interventions in children and adolescents with autism spectrum disorder: a review of recent findings. Current opinion in pediatrics, 23(6), 616-620.

4. Autism and Developmental Disabilities Monitoring Network Surveillance Year 2010 Principal Investigators. 2014. Prevalence of autism spectrum disorder among children aged 8 years-autism and developmental disabilities monitoring network, 11 sites, United States, 2010. Morbidity and Mortality Weekly Report, 63(2), p.1.

5. Mirko Gelsomini. 2016. Supervised Smart Spaces for ASD Children. CHI'16 Workshop Proceedings.

6. Daniel R. Gillette, Gillian R. Hayes, Gregory D. Abowd, Justine Cassell, Rana el Kaliouby, Dorothy Strickland, and Patrice (Tamar) Weiss. 2007. Interactive technologies for autism. In $\mathrm{CHI}$ '07 Extended Abstracts on Human Factors in Computing Systems (CHI EA '07), 2109-2112. http://dx.doi.org/10.1145/1240866.1240960

7. Judith Good, Nicola Yuill, Sarah Parsons, Mark Brosnan, Lisa Austin. 2016. Putting Technology Design into the Hands of the Users with the ASCmeI.T. App. CHI'16 Workshop Proceedings.

8. Francesca Happé, Uta Frith. 2006. The weak coherence account: detail-focused cognitive style in autism spectrum disorders. J. of Autism and Developmental Disorders, 36(1): 5-25.

9. R. Peter Hobson. 1995. Autism and the development of mind. Psychology Press.

10. Leigh J. P., Du J. (2015). Brief Report: Forecasting the Economic Burden of Autism in 2015 and 2025 in the United States. J Autism Dev Disord. 2015 Dec; 45(12), 4135-4139.

11. Kevin Lynch. 1960. The image of the city. Cambridge, MA: MIT Press.

12. Catalin Voss, Nick Haber, Peter Washington, Aaron Kline, Beth McCarthy, Jena Daniels, Azar Fazel, Titas De, Carl Feinstein, Terry Winograd, Dennis Wall. 2016. Designing a Holistic At-Home Learning Aid for Autism. CHI'16 Workshop Proceedings. 